\title{
SECAGEM DE GRÃOS DE CEVADA EM CAMADA FINA: CINÉTICA DE SECAGEM
}

\author{
G. ALBINI ${ }^{1}$, H. PERAZZINI ${ }^{2}$, F. B. FREIRE ${ }^{1}$, J. T. FREIRE $^{1 *}$ \\ ${ }^{1}$ Universidade Federal de São Carlos, Departamento de Engenharia Química \\ ${ }^{2}$ Universidade Federal de Itajubá, Instituto de Recursos Naturais \\ e-mail: freire@ufscar.br
}

\begin{abstract}
RESUMO
No presente trabalho é apresentado um estudo experimental do fenômeno de transferência de massa que se estabelece durante a secagem de grãos de cevada em um secador de leito fixo e camada fina em diferentes condições operacionais. Para fornecer informações sobre o processo de secagem de grãos de cevada, foram realizados experimentos com temperatura do ar de secagem na faixa de $40^{\circ} \mathrm{C}$ a $50^{\circ} \mathrm{C}$, velocidade do ar de $4,0 \mathrm{~m} / \mathrm{s}$ e umidade inicial de 0,16 a 0,36 (base seca).
\end{abstract}

\section{INTRODUÇÃO}

A secagem de grãos é feita com o intuito de se reduzir a atividade da água do material, para que o grão não sofra contaminações microbiológicas, tendo mais durabilidade após a colheita.

Segundo Kunze (2004), a umidade da cevada está sujeita a grandes variações, podendo variar de $12 \%$ a mais de $20 \%$ (base úmida). A cevada armazenada úmida perde sua capacidade germinativa e produz maltes de baixa qualidade. Assim, cevada com conteúdo de umidade superior a $12 \%$ deve passar pelo processo de secagem antes do armazenamento.

Para a secagem, a escolha de um equipamento e condições operacionais adequadas é de fundamental importância para a preservação das qualidades físicas e fisiológicas dos grãos. Os estudos de secagem em leito fixo e camada fina são indispensáveis nos experimentos de cinética de secagem de materiais que se tem pouca informação, auxiliando no entendimento dos fenômenos de transferência de calor e massa.

O objetivo deste trabalho foi determinar, experimentalmente, as curvas de secagem em camada fina de grãos de cevada em função da temperatura do ar de secagem e da umidade inicial do produto e ajustar diferentes modelos matemáticos aos dados experimentais.

\section{MATERIAL E MÉTODOS}

Neste trabalho, foi utilizado um leito delgado de $1 \mathrm{~cm}$ de espessura e diâmetro interno de $10,0 \mathrm{~cm}$ recheado com grãos de cevada (Hordeum vulgare) da variedade Cauê. A espessura do leito foi determinada em experimentos preliminares, realizados com termopares inseridos na entrada e na saída do leito que detectaram uma variação de temperatura menor do que $0,5^{\circ} \mathrm{C}$. A partir destes resultados, a espessura de $1 \mathrm{~cm}$ foi adotada como critério para o estudo da camada fina. Para obter o conteúdo de umidade inicial do grão quando colhido, foi realizada a reumidificação dos grãos de cevada. Uma quantidade de grãos de massa e umidade iniciais conhecidas foi colocada dentro de um pote plástico e foi adicionada a massa calculada de água, de modo que a umidade final fosse pré-estabelecida, através de um balanço de massa (VIEIRA, 2012). 
Com essa metodologia, foram reumidificadas amostras de cevada permitindo obter 3 umidades iniciais diferentes, $\mathrm{X} 1=26,5 \%, \mathrm{X} 2$ $=21,1 \%$ e X3 $=13,6 \%$.

Os estudos de cinética de secagem foram realizados em um secador de leito fixo e camada delgada, onde o meio foi submetido a um escoamento monofásico e ascendente de ar em determinadas condições de velocidade, umidade e temperatura. Nos experimentos, o leito de massa inicial conhecida foi submetido ao processo de secagem e pesado em intervalos de tempo pré-estabelecidos em uma balança semi-analítica (precisão $1 \times 10^{-3} \mathrm{~g}$ ). A massa seca foi obtida mantendo-se o meio em estufa com circulação de ar a $105 \pm 3^{\circ} \mathrm{C}$ por 24 horas. As condições operacionais nas quais os estudos de secagem foram realizados obedecem às seguintes faixas operacionais: 40 $\leq T\left({ }^{\circ} \mathrm{C}\right) \leq 50 ; v(\mathrm{~m} / \mathrm{s})=4,0 ; 2,9 \leq U R(\%) \leq$ 26,$6 ; 13,6 \leq X_{0}(\%$, base úmida $) \leq 26,5$. A umidade relativa do ar dependeu das condições laboratoriais, e foi determinada diretamente por um termo-higrômetro. Os experimentos foram realizados com repetição e os dados mostram-se reprodutíveis, adequados para o estudo da cinética de secagem.

Aos dados experimentais da secagem dos grãos de cevada foram ajustadas equações empíricas e semi-empíricas, frequentemente utilizadas para representação da secagem de produtos agrícolas, apresentadas na Tabela 1.

Além desses quatro modelos semiempíricos, utilizou-se também um modelo teórico, apresentado a seguir.

Tabela 1 - Equações de cinética de secagem.

\begin{tabular}{lc} 
Modelo & \multicolumn{1}{c}{ Equação } \\
\hline Page & $M R=\exp \left(-K_{P} t^{n_{P}}\right)$ \\
Lewis & $M R=\exp \left(-K_{L} t\right)$ \\
Henderson e Pabis & $M R=a \exp \left(-K_{H P} t\right)$ \\
Overhults & $M R=\exp \left[-\left(K_{O} t\right)^{n_{O}}\right]$ \\
\hline
\end{tabular}

$\mathrm{O}$ adimensional de umidade $(M R)$ foi obtido pela definição:

$$
M R=\frac{\bar{X}_{t}-X_{e q}}{X_{0}-X_{e q}}
$$

onde $\bar{X}_{t}$ é a umidade média em determinado tempo do processo, $X_{e q}$ é a umidade de equilíbrio dinâmico e $X_{0}$ é a umidade inicial da amostra.

A difusividade efetiva, estimada a partir do modelo difusivo, é um parâmetro mássico tradicionalmente obtido com abordagem teórica do problema a partir de uma equação similar a da Segunda Lei de Fick (EFREMOV et al., 2008), tendo a $D_{\text {ef }}$ no lugar da difusividade ordinária.

Assumindo a difusividade efetiva e as propriedades do sólido constantes, a célula de secagem aproximada para uma placa plana de espessura L e infinita, o encolhimento do material desprezível e a transferência de massa unidimensional (direção z), temos:

$$
\frac{\partial X}{\partial t}=D_{e f} \frac{\partial^{2} X}{\partial z^{2}}
$$

A Equação 2 está sujeita às seguintes condições inicial e de contorno:

$$
\begin{aligned}
& \text { C.I }: X=X_{0} \text { em t }=0,0<\mathrm{z}<\mathrm{L} \\
& \text { C.C. } 1:\left(\frac{\partial X}{\partial z}\right)=0 \text { em } \mathrm{z}=0, \mathrm{t}>0 \\
& \text { C.C. } 2: X=X_{e q} \text { em } \mathrm{z}=\mathrm{L}, \mathrm{t}>0
\end{aligned}
$$

A Equação 2, submetida às condições apresentadas, tem sua solução analítica obtida pelo método de separação de variáveis (CRANK, 1975): 


$$
\begin{aligned}
& \frac{\mathrm{X}_{\mathrm{t}}-\mathrm{X}_{\mathrm{eq}}}{\mathrm{X}_{0}-\mathrm{X}_{\mathrm{eq}}} \\
& =\frac{4}{\pi} \sum_{n=0}^{\infty} \frac{(-1)^{n}}{(2 \mathrm{n}+1)} \cos \left[\left(\frac{2 n+1}{2 L}\right) \pi z\right] \\
& \exp \left[-\frac{(2 n+1)^{2}}{4 L^{2}} \pi^{2} D_{e f} t\right]
\end{aligned}
$$

A umidade média é obtida pela Equação 4, pois em estudos de camada fina o que se obtém é a concentração média espacial de umidade da célula de secagem ao longo do tempo.

$$
\bar{X}(t)=\frac{1}{L} \int_{0}^{L} X d z
$$

Substituindo a Equação 3 na Equação 4 e integrando, a umidade adimensional do meio homogêneo (MR) em função do tempo é dada por:

$$
\begin{aligned}
& M R=\frac{\bar{X}(t)-X_{e q}}{X_{0}-X_{e q}} \\
& =\frac{8}{\pi^{2}} \sum_{n=0}^{\infty} \frac{1}{(2 n+1)^{2}} \\
& \quad \exp \left[-\left(n+\frac{1}{2}\right)^{2} \pi^{2} \frac{D_{e f}}{L^{2}} t\right]
\end{aligned}
$$

Para a estimação dos parâmetros que constituem os modelos matemáticos utilizados neste trabalho foi empregado um pacote computacional que leva a uma melhor aproximação entre as curvas teóricas e experimentais através da minimização de uma função objetivo. Utilizou-se a minimização do quadrado dos desvios como critério para todas as estimativas, implementadas em rotina computacional desenvolvida em linguagem MatLab® (R2007b, Mathworks).

Os critérios estatísticos utilizados para avaliar os ajustes foram o coeficiente de determinação $\left(R^{2}\right)$, erro parcial médio $(M S E)$, raiz quadrada do erro médio ( $R M S E)$ e chi- quadrado $\left(\chi^{2}\right)$, os três últimos são determinados pelas equações a seguir.

$$
\begin{aligned}
& M S E=\frac{1}{N} \sum_{i=1}^{N}\left(x_{\text {pred }, i}-x_{\text {exp }, i}\right) \\
& R M S E=\sqrt{\frac{1}{N} \sum_{i=1}^{N}\left(x_{\text {pred }, i}-x_{\text {exp }, i}\right)^{2}} \\
& \chi^{2}=\frac{1}{N-p} \sum_{i=1}^{N}\left(x_{\text {pred }, i}-x_{\text {exp }, i}\right)^{2}
\end{aligned}
$$

\section{REULTADOS E DISCUSSÃO}

Na Figura 1 são apresentados os dados experimentais da cinética de secagem de grãos de cevada com diferentes conteúdos de umidade inicial que passaram pelo processo de reumidificação.

Verifica-se nas Figuras 1 (a) a 1 (c) que o efeito dos diferentes conteúdos de umidade inicial é mais significativo na região intermediária, sendo observada uma diminuição na taxa de secagem para a umidade X3 $(0,16)$ após 100 minutos do início do experimento, quando comparado com as taxas de secagem de X2 $(0,27)$ e X1 $(0,36)$, para as três temperaturas. Isto se deve ao fato de que quanto menor a quantidade de água no grão, mais ligada ela está, consequentemente a taxa diminui porque é necessário empregar mais energia para removê-la do que em ocasiões com umidade maior.

Nas Figuras 2 (a) a 2 (c) o aumento da temperatura influencia $\mathrm{o}$ processo de secagem. Observa-se que o aumento da temperatura de $40^{\circ} \mathrm{C}$ para $50^{\circ} \mathrm{C}$ promoveu a diminuição da umidade em um tempo menor, pois ocorre um aumento na quantidade de energia térmica transferida do ar para o 
sólido, facilitando a transferência de umidade

do

sólido

para

o

ar.

Figura 1 - Cinética de secagem para diferentes conteúdos de umidade inicial: (a) $\mathrm{T}=40^{\circ} \mathrm{C}$; (b) $\mathrm{T}=45^{\circ} \mathrm{C}$; (c) $\mathrm{T}$ $=50^{\circ} \mathrm{C}$.

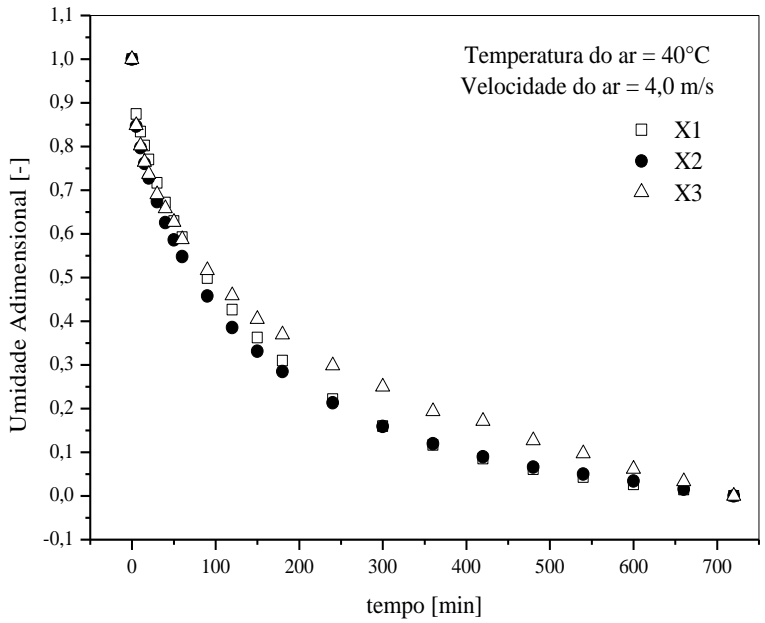

(a)

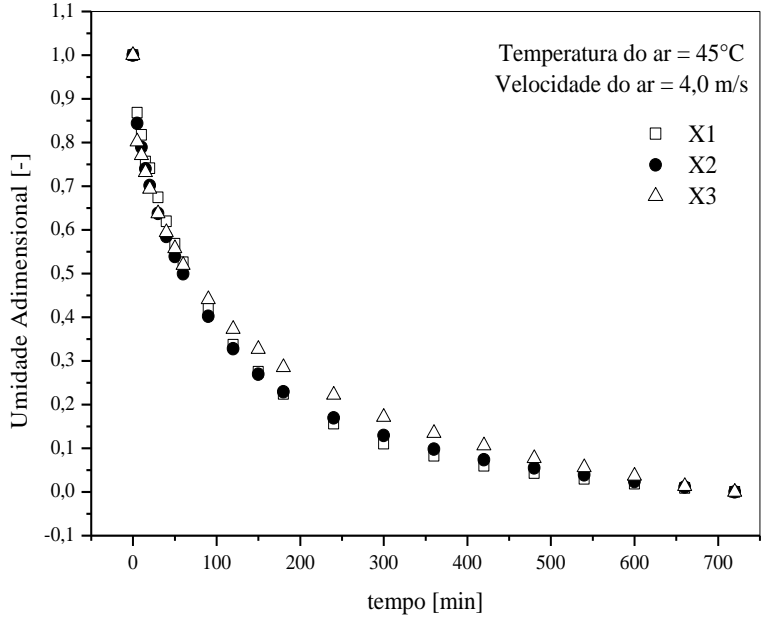

(b)

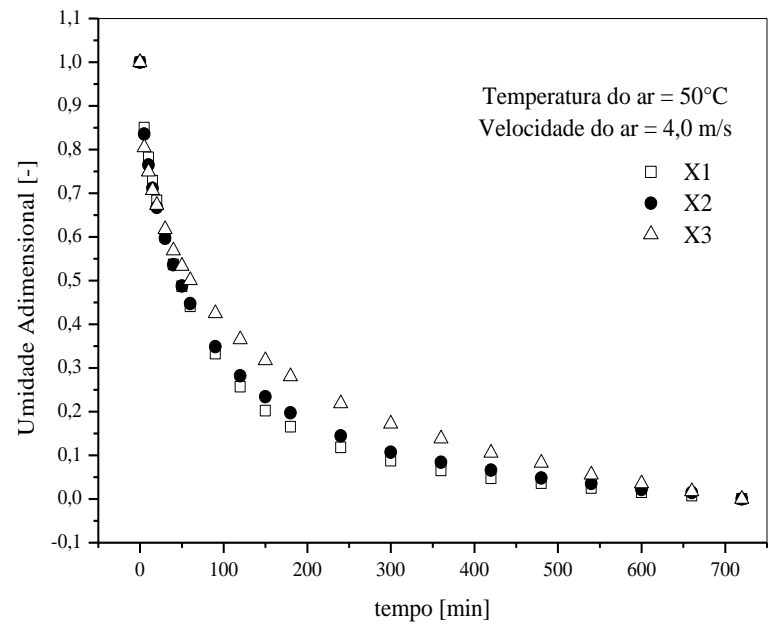

(c)

A Figura 3 apresenta, como exemplo, resultados da comparação da resposta fornecida pela equação de Page e equação de Overhults confrontados com os dados de secagem obtidos experimentalmente. Observa-se que ambas as equações coincidem e representam bem os dados experimentais, ocorrendo uma superestimação pelas equações ao final da secagem. Na Tabela 2 estão representados os valores dos parâmetros estatísticos analisados para as cinco equações ajustadas.

O coeficiente de determinação $\left(\mathrm{R}^{2}\right)$ foi o primeiro critério estatístico para selecionar a melhor correlação. Além do $\mathrm{R}^{2}$, o erro parcial médio (MSE), a raiz quadrada do erro médio (RMSE) e o chi-quadrado $\left(\chi^{2}\right)$, foram usados para indicar um bom ajuste dos modelos aos dados experimentais de secagem. Quanto maior o valor de $\mathrm{R}^{2}$ e menores os valores de 
MSE, RMSE e $\chi^{2}$, melhor o ajuste (PEREIRA, 2010). Assim, analisando os valores obtidos para os critérios estatísticos, os modelos Difusivo, de Page e Overhults obtiveram os melhores ajustes aos dados experimentais.

Figura 2 - Cinética de secagem: (a) X1 =0,36 ( $\mathrm{g}_{\text {água }} / \mathrm{g}_{\text {sól. seco }}$ ); (b) X2 =0,27( $\mathrm{g}_{\text {água }} / \mathrm{g}_{\text {sól. seco }}$ ) e (c) X3 = 0,16 ( $\left.\mathrm{g}_{\text {água }} / \mathrm{g}_{\text {sól. seco }}\right)$.

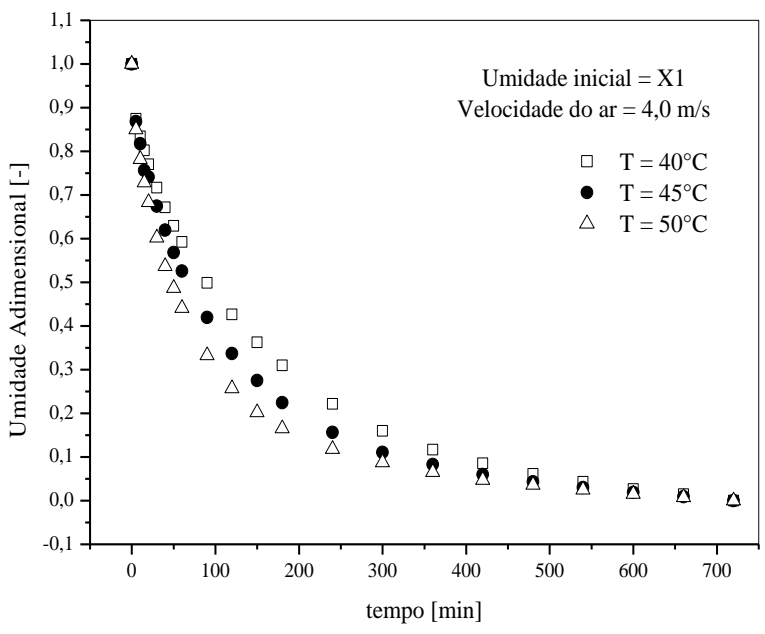

(a)

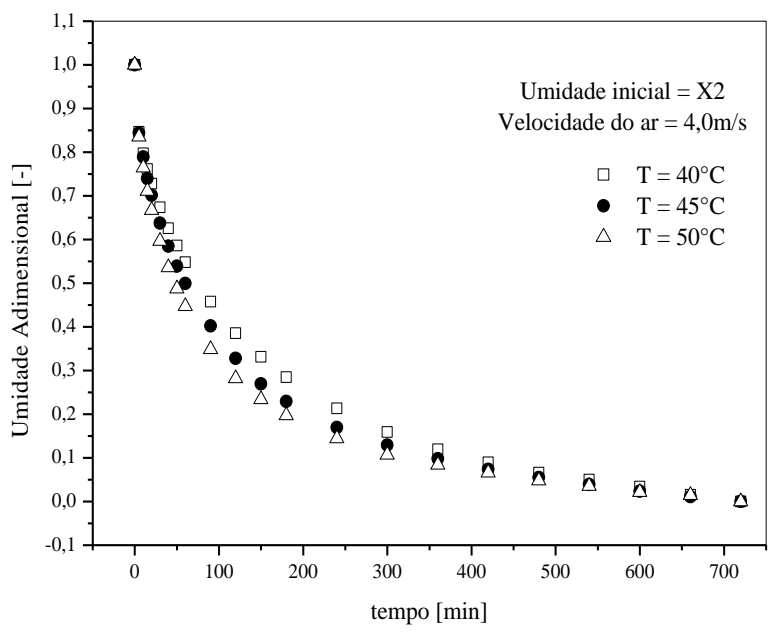

(b)

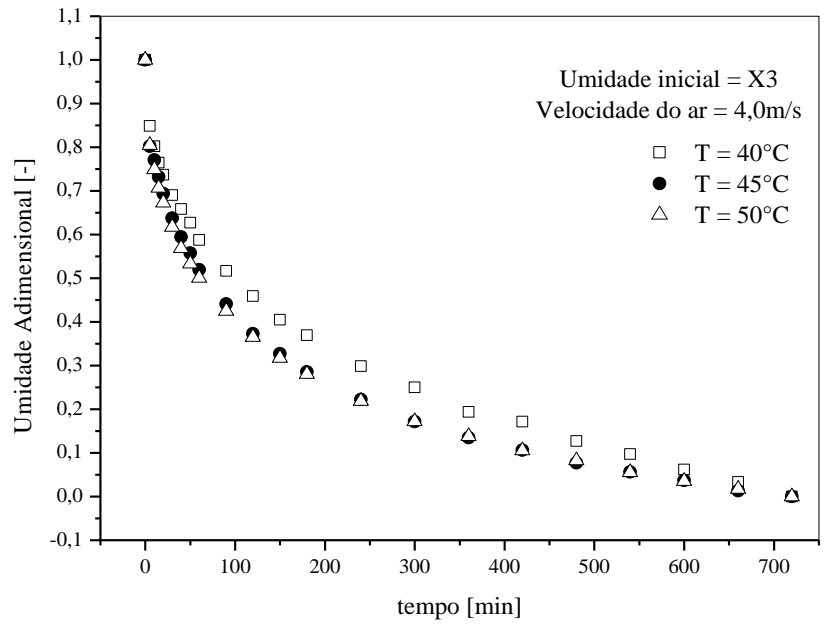

(c)

Com os dados apresentados na Tabela 2, verifica-se que o Modelo de Page e o Modelo de Overhults apresentam os mesmos valores para o coeficiente de determinação, para o erro parcial médio, para a raiz quadrada do erro médio e para o chiquadrado, sendo indicados para representar a cinética de secagem dos grãos de cevada. Este resultado era esperado, pois com um simples rearranjo das equações e uma parametrização permitem verificar que as equações são equivalentes. Na literatura, o Modelo de Overhults é também denominado como Modelo de Page Modificado. 
Figura 3 - Curvas de secagem ajustadas pelo Modelo de Page e Modelo de Overhults.

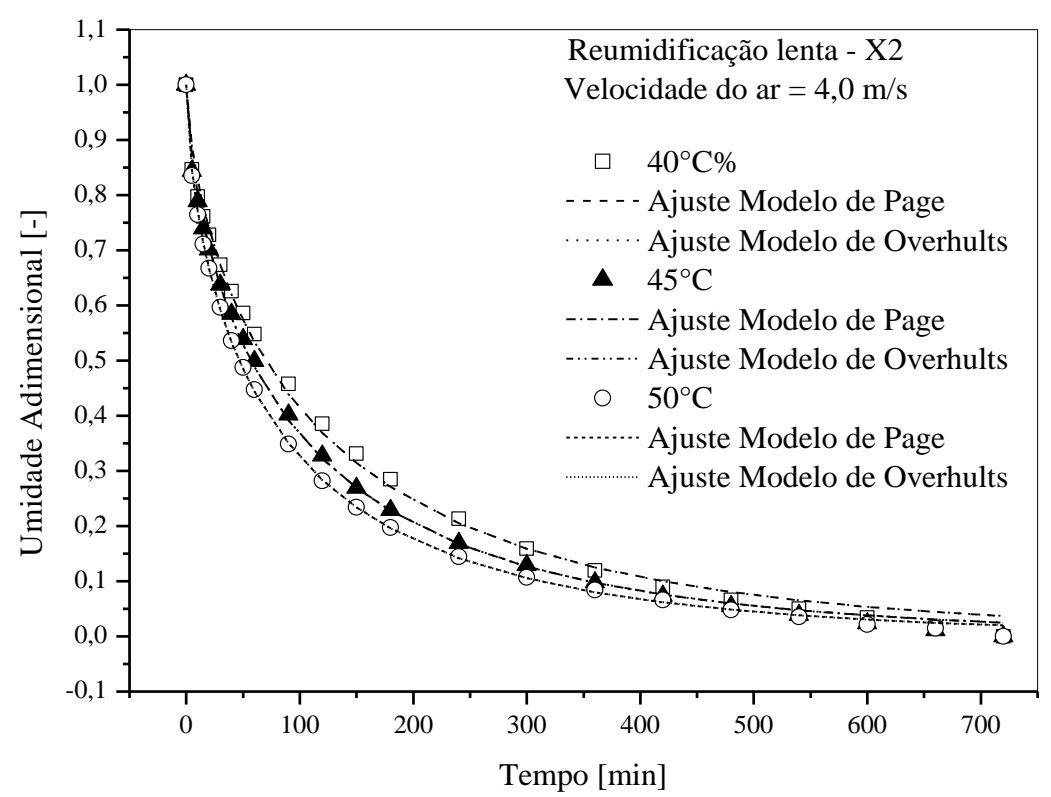

Tabela 2 - Parâmetros estatísticos dos modelos ajustados.

\begin{tabular}{|c|c|c|c|c|c|c|c|c|c|c|c|c|c|}
\hline \multirow[b]{3}{*}{$\begin{array}{l}\text { Umidade } \\
\text { (b.s.) }\end{array}$} & \multirow[b]{3}{*}{ Modelo } & \multicolumn{12}{|c|}{ Temperatura } \\
\hline & & \multicolumn{4}{|c|}{$40^{\circ} \mathrm{C}$} & \multicolumn{4}{|c|}{$45^{\circ} \mathrm{C}$} & \multicolumn{4}{|c|}{$50^{\circ} \mathrm{C}$} \\
\hline & & $\mathbf{R}^{2}$ & MSE & RMSE & $\chi^{2}$ & $\mathbf{R}^{2}$ & MSE & RMSE & $\chi^{2}$ & $\mathbf{R}^{2}$ & MSE $F$ & RMSE & $\chi^{2}$ \\
\hline \multirow{5}{*}{$\mathrm{X} 1$} & Difusivo & 0,9997 & 0,0035 & 0,0052 & $2,84 \mathrm{E}-05$ & 0,9991 & 0,0082 & 0,0095 & $9,52 \mathrm{E}-05$ & 0,9960 & 0,0157 & $7 \quad 0,0198$ & 4,11E-04 \\
\hline & Page & 0,9962 & 0,0169 & 0,0199 & $4,38 \mathrm{E}-04$ & 0,9986 & 0,0094 & 0,0120 & $1,59 \mathrm{E}-04$ & 0,9997 & 0,0048 & $8 \quad 0,0056$ & $3,39 \mathrm{E}-05$ \\
\hline & Lewis & 0,9709 & 0,0468 & 0,0553 & $3,20 \mathrm{E}-03$ & 0,9723 & 0,0456 & 0,0537 & $3,00 \mathrm{E}-03$ & 0,9672 & 0,0479 & $9 \quad 0,0567$ & $3,40 \mathrm{E}-03$ \\
\hline & $\begin{array}{c}\text { Henderson } \\
\text { e Pabis }\end{array}$ & 0,9803 & 0,0190 & 0,0279 & $8,56 \mathrm{E}-04$ & 0,9928 & 0,0231 & 0,0312 & $1,00 \mathrm{E}-03$ & 0,9909 & 0,0305 & $5 \quad 0,0384$ & $1,60 \mathrm{E}-03$ \\
\hline & Overhults & 0,9962 & 0,0169 & 0,0199 & $4,38 \mathrm{E}-04$ & 0,9986 & 0,0094 & 0,0120 & $1,59 \mathrm{E}-04$ & 0,9997 & 0,0048 & $8 \quad 0,0056$ & $3,39 \mathrm{E}-05$ \\
\hline \multirow{5}{*}{$\mathrm{X} 2$} & Difusivo & 0,9961 & 0,0175 & 0,0194 & $3,93 \mathrm{E}-04$ & 0,9943 & 0,0203 & 0,0233 & $5,70 \mathrm{E}-04$ & 0,9897 & 0,0269 & $9 \quad 0,0308$ & $9,91 \mathrm{E}-04$ \\
\hline & Page & 0,9963 & 0,0155 & 0,0188 & $3,90 \mathrm{E}-04$ & 0,9987 & 0,0083 & 0,0111 & $1,37 \mathrm{E}-04$ & 0,9996 & 0,0041 & $1 \quad 0,0061$ & 4,09E-05 \\
\hline & Lewis & 0,9453 & 0,0618 & 0,0723 & $5,50 \mathrm{E}-03$ & 0,9487 & 0,0602 & 0,0698 & $5,10 \mathrm{E}-03$ & 0,9432 & 0,0618 & $8 \quad 0,0721$ & $5,40 \mathrm{E}-03$ \\
\hline & $\begin{array}{l}\text { Henderson } \\
\text { e Pabis }\end{array}$ & 0,9895 & 0,0283 & 0,0390 & $1,70 \mathrm{E}-03$ & 0,9885 & 0,0321 & 0,0423 & $2,00 \mathrm{E}-03$ & 0,9831 & 0,0383 & 30,0477 & $2,50 \mathrm{E}-03$ \\
\hline & Overhults & 0,9963 & 0,0155 & 0,0188 & $3,90 \mathrm{E}-04$ & 0,9987 & 0,0083 & 0,0111 & $1,37 \mathrm{E}-04$ & 0,9996 & 0,0041 & 10,0061 & $4,09 \mathrm{E}-05$ \\
\hline \multirow{5}{*}{$\mathrm{X} 3$} & Difusivo & 0,9872 & 0,0295 & 0,0295 & $1,20 \mathrm{E}-03$ & 0,9828 & 0,0338 & 0,0387 & $1,60 \mathrm{E}-03$ & 0,9731 & 0,0415 & $5 \quad 0,0473$ & $2,30 \mathrm{E}-03$ \\
\hline & Page & 0,9896 & 0,0242 & 0,0299 & $9,80 \mathrm{E}-04$ & 0,9938 & 0,0179 & 0,0231 & $5,88 \mathrm{E}-04$ & 0,9942 & 0,0176 & $6 \quad 0,0220$ & $5,31 \mathrm{E}-04$ \\
\hline & Lewis & 0,9052 & 0,0778 & 0,0903 & $8,50 \mathrm{E}-03$ & 0,9058 & 0,0773 & 0,0905 & $8,60 \mathrm{E}-03$ & 0,8847 & 0,0846 & $6 \quad 0,0980$ & $1,01 \mathrm{E}-02$ \\
\hline & $\begin{array}{c}\text { Henderson } \\
\text { e Pabis }\end{array}$ & 0,9761 & 0,0334 & 0,0457 & $2,30 \mathrm{E}-03$ & 0,9788 & 0,0339 & 0,0490 & $2,60 \mathrm{E}-03$ & 0,9758 & 0,0393 & 30,0548 & $3,30 \mathrm{E}-03$ \\
\hline & Overhults & 0,9896 & 0,0242 & 0,0299 & $9,80 \mathrm{E}-04$ & 0,9938 & 0,0179 & 0,0231 & $5,88 \mathrm{E}-04$ & 0,9942 & 0,0176 & $6 \quad 0,0220$ & $5,31 \mathrm{E}-04$ \\
\hline
\end{tabular}


$\mathrm{Na}$ Figura 4 é apresentada uma comparação típica entre os dados observados e os preditos pelo Modelo Difusivo. O Modelo Difusivo foi resolvido pelo método de separação de variáveis (CRANK, 1975). Foram utilizados 100 termos na série, Equação 5, o que proporciona um erro de $0,01 \%$ no instante inicial de secagem $(\mathrm{t}=0)$. A difusividade efetiva foi estimada com base nos critérios dos mínimos quadrados.

Com base nos resultados apresentados, observa-se que apesar dos valores para os coeficientes de determinação serem superiores a 0,9 , o ajuste proporcionado pelo Modelo Difusivo com $D_{e f}$ constante não é satisfatório, subestimando os dados na etapa intermediária até o término do processo de secagem.
Possivelmente, os desvios encontrados entre os dados observados e preditos podem estar relacionados com as considerações feitas para solução do modelo. Dentre as considerações temos: encolhimento dos grãos desprezível e difusividade efetiva como constante. Com a caracterização física do material em função da umidade verificou-se que ocorre encolhimento e no Modelo Difusivo com $D_{e f}$ constante, os dados experimentais são ajustados considerando um valor médio do coeficiente difusivo, mas este parâmetro pode assumir diferentes comportamentos, sendo uma função, principalmente, da umidade e da temperatura do material (PERAZZINI et al., 2013).

Figura 4 - Dados de cinética de secagem observados e preditos pelo modelo difusivo.

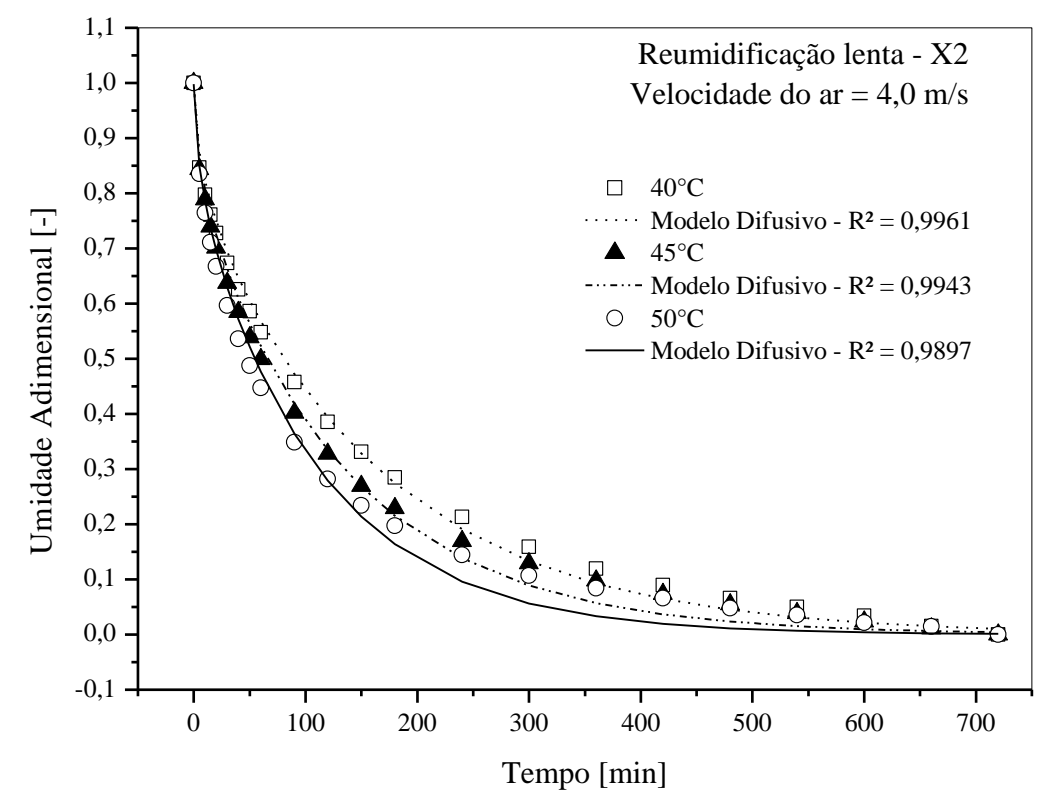

A Figura 5 mostra a dependência da difusividade efetiva $\left(D_{\text {ef }}\right)$ com a temperatura e a umidade inicial dos grãos de cevada. Khatchatourian (2012) verificou para grãos de soja que o valor da difusividade efetiva elevase quando os valores de temperatura e umidade inicial também são elevados e Doymaz (2005) estudando a cinética de secagem de feijão verde, verificou que a difusividade efetiva possui um comportamento linear crescente com a temperatura.

Ao ajustar um comportamento linear crescente com a temperatura para os valores de $D_{\text {ef }}$ estimados pelo Modelo Difusivo, Figura 5, observa-se que este ajuste não é satisfatório, pois os valores de $\mathrm{D}_{\text {ef }}$ para $\mathrm{X} 1$ (26,5 b.u.) decrescem quando comparados aos 
valores de $\mathrm{D}_{\text {ef }}$ para X2 (21,1 b.u.). Fato que pode ser explicado devido ao encolhimento que ocorre no leito de material durante o processo de secagem ser mais significativo para X1 que para X2. O encolhimento é considerado desprezível pelo Modelo Difusivo utilizados para nas estimativas de $D_{\text {ef. }}$.

Figura 5 - Difusividade efetiva em função da temperatura para diferentes conteúdos iniciais de umidade.

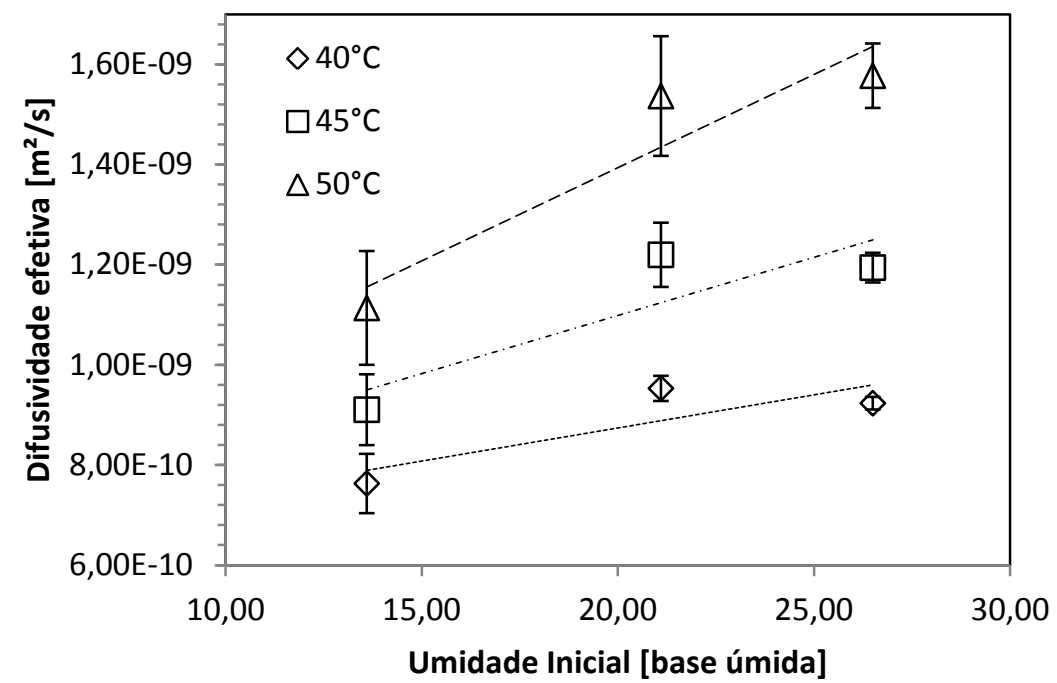

Neste trabalho, como o coeficiente de difusão $\left(\mathrm{D}_{\mathrm{ef}}\right)$ é tratado como uma difusividade efetiva, esta consideração permite avaliar a dependência da difusividade efetiva com a temperatura através de uma correlação do tipo Arrhenius:

$$
D_{e f}=D_{0} \exp \left(-\frac{E a}{R \bar{T}}\right)
$$

A Equação 9 é fortemente não linear e utilizar apenas três dados de temperatura para estimar os parâmetros pode não ser o mais adequado, mas como para este trabalho temos apenas estes dados. A Figura 6 apresenta a linearização da Equação 9, a inclinação da curva de correlação do tipo Arrhenius fornece a relação $(\mathrm{Ea} / \mathrm{R})$ enquanto sua intersecção com o eixo das ordenadas indica o valor de $\mathrm{D}_{0}$. Segundo Kashaninejad et al. (2007), termodinamicamente, a energia de ativação (Ea) representa a energia necessária para o rompimento da barreira que as moléculas de água encontram, durante $\mathrm{o}$ processo de secagem, ao migrarem do interior para a superfície do produto.

$\mathrm{Na}$ Tabela 3 estão apresentados os resultados dos ajustes mostrados na Figura 6. Os valores da energia de ativação se mostram condizentes com a literatura, segundo Zogzas et al. (1996) os valores da energia de ativação para produtos agrícolas varia entre 13 e 110 $\mathrm{kJ} / \mathrm{mol}$

Tabela 3 - Parâmetros da correlação do tipo Arrhenius.

\begin{tabular}{lccc}
\hline Parâmetros & X1 & X2 & X3 \\
\hline $\mathrm{D}_{0}\left[\mathrm{~m}^{2} / \mathrm{s}\right]$ & $3,00 \mathrm{E}-02$ & $4,87 \mathrm{E}-03$ & $1,53 \mathrm{E}-04$ \\
$\mathrm{Ea}[\mathrm{J} / \mathrm{mol}]$ & 45045,47 & 40215,57 & 31807,12 \\
$\mathrm{R}^{2}$ & 0,999 & 0,9999 & 0,9978 \\
\hline
\end{tabular}


Figura 6 - Correlação do tipo Arrhenius entre o coeficiente de difusividade efetiva e temperatura.

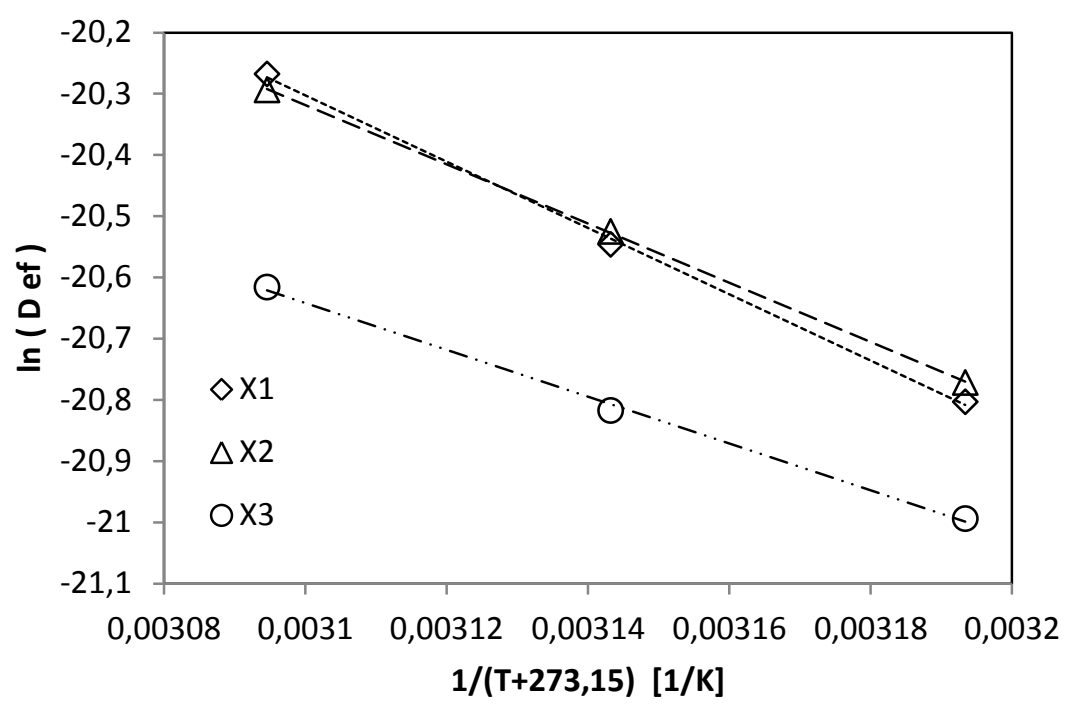

\section{CONCLUSÃO}

Os experimentos de cinética de secagem em secador de leito fixo e camada fina forneceram dados de umidade em função do tempo, em que, como esperado, se verificou que a temperatura do ar tem influência significativa na secagem dos grãos de cevada.

A partir da discriminação das principais equações de secagem empíricas e semiempíricas, com a aplicação de inferências estatísticas, a equação de Page e de Overhults apresentaram o melhor ajuste aos dados de umidade em função do tempo.

A cinética de secagem ajustada ao Modelo Difusivo apresentou coeficiente de determinação superior a 0,9 , mas o ajuste não foi satisfatório, pois subestimou os resultados da etapa intermediária ao término do processo. Estes desvios, possivelmente, estão relacionados ao fato de existir encolhimento do grão e por considerar a difusividade efetiva constante, já que se verificou um aumento da difusividade efetiva com o aumento da umidade do grão e da temperatura do ar de secagem.

\section{NOMENCLATURA}

Ea - energia de ativação

$\left[\mathrm{Jmol}^{-1}\right]$

a - parâmetro da equação de Henderson e Pabis

$\mathrm{D}_{\mathrm{ef}} \quad$ - Difusividade efetiva $\left[\mathrm{m}^{2} / \mathrm{s}\right]$

$D_{0} \quad$ - parâmetro da correlação de Arrhenius

$K_{0}$ - parâmetro da correlação de Arrhenius

$K_{L} \quad$ - constante cinética do modelo de Lewis

$K_{P} \quad$ - constante cinética do modelo de Page

$K_{H P} \quad$ - constante cinética do modelo de Henderson e Pabis

$K_{O} \quad$ - constante cinética do modelo de Overhults

$\mathrm{n}_{\mathrm{p}} \quad$ - constante cinética da equação de Page

$\mathrm{n}_{\mathrm{o}} \quad$ - constante cinética da equação de Overhults

$R \quad$ - constante universal dos $\quad\left[\mathrm{Jmol}^{-1} \mathrm{~K}^{-1}\right]$ gases ideais

t - tempo

$\mathrm{T}$ - temperatura do ar

$\bar{T} \quad$ - temperatura 


$$
\begin{aligned}
& \text { 6) } \\
& \text { v - velocidade do ar } \\
& \text { UR -umidade relativa } \\
& \text { bs - base seca } \\
& \text { bu - base úmida } \\
& \text { exp - experimental } \\
& \text { pred - predito }
\end{aligned}
$$

\section{REFERÊNCIAS BIBLIOGRÁFICAS}

BARROZO, M. A. S. Transferência de calor e massa entre ar e sementes de soja em leito deslizante e escoamentos cruzados. 1995. 163 p. Tese (Doutorado em Engenharia Química) - Departamento de Engenharia Química, Universidade Federal de São Carlos, São Carlos, SP, 1995

CRANK, J. The mathematics of diffusion. Claredon Press: Oxford, 1975.

DOYMAZ, I. Drying behavior of green beans. Journal of Food Engineering. 2005. Vol. 69, p. 161-165.

EFREMOV, G., MARKOWSKI, M., BIALOBRZEWSKI, I., ZIELINSKA, M. Approach to calculation time-dependent moisture diffusivity for thin layered biological materials. Int. Commun. Heat Mass Transf., 2008. Vol. 35, p. 1069-1072.

KASHANINEJAD, M.; MORTAZAVI, A.; SAFEKORDI, A.; TABIL, L.G. Thin-layer drying characteristics and modeling of pistachio nuts. Journal of Food Engineering. 2007. Vol. 78, p. $98-108$.

KHATCHATOURIAN,O. A. Experimental study and mathematical model for soya bean drying in thin layer. Biosystems engineering. Vol. 113. 2012. p. 54 - 64.
KUNZE, W. Technology Brewing and Malting. Berlin: Versuchs und Lehranstalt für Brauerei in Berlin (VLB). 2004.

PERAZZINI, H., FREIRE, F. B., FREIRE, J. T.Estudo da difusividade efetiva na secagem de meios porosos como parâmetro constante ou variável. Anais do XXXVI Congresso Brasileiro de Sistemas Particulados (ENEMP). 2013.

PEREIRA, A. V. Caracterização e secagem de sementes de painço (Setaria italica L.). 2010. 92 p. Dissertação (Mestrado em Engenharia Química) - Departamento de Engenharia Química, Universidade Federal de São Carlos, São Carlos, SP, 2010.

VIEIRA, G. N. A. Caracterização e secagem de grãos de linhaça marrom (Linum usitatissimum $L$.) em leito fixo e fluidizado. 2012. 82 p. Dissertação (Mestrado em Engenharia Química) - Departamento de Engenharia Química, Universidade Federal de São Carlos, São Carlos, SP, 2012.

ZOGZAS, N. P.; MAROULIS, Z. B.; MARINOS-KOURIS, D. Moisture diffusivity data compilation in foodstuffs. Drying Technology. 1996. Vol. 14, n. 10. p. $2225-$ 2253.

\section{AGRADECIMENTOS}

Ao Programa de Pós-Graduação em Engenharia Química (PPGEQ - UFSCar), ao Conselho Nacional de Desenvolvimento Científico e Tecnológico (CNPq) e à Coordenação de Aperfeiçoamento de Pessoal de Nível Superior (CAPES) pelo apoio financeiro essencial para o desenvolvimento deste trabalho. 